\title{
PDF Uncertainties at Hadron Colliders
}

\author{
Aidan Robson ${ }^{* \dagger}$ \\ Department of Physics and Astronomy, University of Glasgow, Glasgow G12 8QQ, UK \\ E-mail: a.robsonephysics.gla.ac.uk
}

An overview is given of parton distribution function (PDF) uncertainties at the Tevatron. Recent results that provide PDF-constraining power are reviewed and a description given of recent measurements that find a significant contribution from PDFs to their total systematic uncertainties.

Diffraction 06, International Workshop on Diffraction in High-Energy Physics September 5-10, 2006

Adamantas, Milos Island, Greece

${ }^{*}$ Speaker.
${ }^{\dagger}$ For the CDF and D0 Collaborations. 


\section{Introduction}

The Tevatron experiments CDF and D0 have wide-ranging physics programmes, making precision measurements of the Standard Model and searching for new physics. From the point of view of measuring the content of the proton, data from the Tevatron act as an important pivot between the relatively low $Q^{2}$ probed at HERA, the source of most of the constraints on parton densities, and the high scales that will be accessible at the LHC. After a brief introduction to the CDF and D0 experiments, this contribution discusses the quantifying of PDF uncertainties, gives a brief insight into some important measurements that find significant PDF uncertainties, and then reviews recent results that have potential to act as further constraints on PDF fits.

\section{The CDF and D0 experiments}

$\mathrm{CDF}$ and D0 are general-purpose detectors, designed to trigger on and accurately reconstruct electrons, muons, charged tracks, jets, and the imbalance in measured energy that comes from neutrinos. CDF has excellent tracking capabilities provided by a drift chamber covering $|\eta|<1$, and extended forward by silicon detectors. A muon system provides coverage for $|\eta|<1.5$, and calorimetry extends to $|\eta|<3$. D0 has excellent muon coverage for $|\eta|<2$ and calorimetry for $|\eta|<4$. The D0 fibre tracker covers the region $|\eta|<1.8$.

\section{PDF uncertainties}

\subsection{Quantifying PDF uncertainities}

PDFs are parameterised fits to many sets of data collected by deep inelastic scattering, fixedtarget and hadron collider experiments. Several groups provide PDF sets, and among the most widely used are those from the CTEQ and MRST collaborations $[1,2]$. As an example, the functional form used by the CTEQ collaboration is:

$$
x f_{a}\left(x, Q_{0}\right)=A_{0} x^{A_{1}} \cdot(1-x)^{A_{2}} \cdot e^{A_{3} x} \cdot\left(1+A_{4} x\right)^{A_{5}},
$$

where $a$ are combinations of $\mathrm{u}-, \mathrm{d}-, \overline{\mathrm{u}}$ - and $\overline{\mathrm{d}}$-quarks and the gluon, and $f_{j}(x, Q)$ is the probability of finding parton $j$ carrying a fraction $x$ of the proton's momentum, when it is probed at scale $Q$. Of the resulting 30 parameters $A_{i}^{a}, 10$ are fixed. The remaining free parameters are determined at a low scale $Q_{0}=1.3 \mathrm{GeV}$, and QCD evolution equations are used to extract values for the parton densities at the high values of $Q^{2}$ of interest at the Tevatron.

A relatively recent development is the provision of PDF 'error' sets that encode uncertainties in and disagreements between the fitted data [3,4]. Eigenvectors are formed in $A_{i}$-space -20 eigenvectors for CTEQ and 15 for MRST - and for each eigenvector, two complete PDF sets are provided corresponding to the changes in each direction of that eigenvector that increase the $\chi^{2}$ of the overall fit by a given amount from its minimum. The CTEQ collaboration provides error PDF sets at $\Delta \chi^{2}=100$ from its best fit, and the MRST collaboration uses $\Delta \chi^{2}=50$.

Using these error PDF sets in the same way as the best-fit sets, as input to a cross-section calculation or event generator and detector simulation, enables a determination of the PDF uncertainty for a particular measurement. 


\subsection{Current limitations arising from PDF uncertainties}

$\mathbf{W}$ and $\mathbf{Z}$ cross-sections. Some of the earliest high-precision high- $p_{T}$ measurements to come from CDF during Run 2 of the Tevatron were the $\mathrm{W}$ and $\mathrm{Z}$ cross-section measurements [5]. The uncertainties on these measurements are dominated by the $6 \%$ luminosity uncertainty common to all Tevatron cross-section measurements, but other than that the systematic uncertainties are very well under control, to better than $2 \%$. One of the largest contributions to the systematic uncertainty is the PDF uncertainty, at $\sim 1 \%$.

The ratio of $\mathrm{W}$ to $\mathrm{Z}$ cross-sections is of interest because several fundamental parameters of the Standard Model can be extracted from it, and experimentally many of the uncertainties cancel when taking the ratio, including the luminosity uncertainty which cancels entirely. However in the CDF measurement there remains a significant PDF uncertainty in the ratio and therefore in the extracted value of the $\mathrm{W}$ boson width $\Gamma(\mathrm{W})=2092 \pm 42 \mathrm{MeV}$, with a $0.6 \%$ contribution to the total uncertainty coming from PDFs.

In this situation, one approach is to design a measurement specifically to minimise the PDF uncertainties. It was realised that the non-cancellation of the PDF uncertainty in the ratio measurement is largely a result of the very different rapidity distributions of $\mathrm{W}$ and $\mathrm{Z}$ events accepted in the analysis. In a new analysis that is ongoing, by making the $\mathrm{W}$ and $\mathrm{Z}$ event selections identical (requiring only one lepton even for $\mathrm{Z}$ events) and determining the ratio of cross-sections by fitting observed kinematic distributions with a combination of $\mathrm{W}$ and $\mathrm{Z}$ simulated events, the PDF uncertainty is expected to be reduced to $0.3 \%$.

Top pair production. The current combined CDF t-quark cross-section measurement, using $760 \mathrm{pb}^{-1}$ of data, is $\sigma(\mathrm{p} \overline{\mathrm{p}} \rightarrow \mathrm{t} \overline{\mathrm{t}})=\left(7.3 \pm 0.5_{\text {stat }} \pm 0.6_{\text {sys }} \pm 0.4_{\text {lum }}\right) \mathrm{pb}$ [6]. However the best theoretical calculation gives $\sigma(\mathrm{p} \overline{\mathrm{p}} \rightarrow \mathrm{t} \overline{\mathrm{t}})=6.7_{-0.9}^{+0.7} \mathrm{pb}$ [7], which includes an uncertainty of $\sim 10 \%$ from PDFs. In this case, imperfect knowledge of the proton content is limiting our ability to test the Standard Model.

W boson mass. The neutrino produced in the leptonic decay of a $\mathrm{W}$ boson escapes the detector, so the $\mathrm{W}$ boson mass is measured by fitting to templates of lepton momentum transverse to the beam direction, to the measured energy imbalance in the transverse plane or to a mass $m_{T}$ constructed only from transverse quantities. The $\mathrm{W}$ mass measurement is still being finalised but $\mathrm{CDF}$ has evaluated the uncertainties for its $200 \mathrm{pb}^{-1}$ dataset. The total uncertainty is projected to be $76 \mathrm{MeV}$, which is dominated by the lepton and recoil energy scales and resolution. However these leading systematic uncertainties scale roughly with luminosity and will be correspondingly reduced in the next iteration of the analysis. In contrast, the contribution to the uncertainty of $15 \mathrm{MeV}$ from the PDFs will not reduce and will be a significant component of the total expected uncertainty of $40 \mathrm{MeV}$ for Run 2 .

\section{PDF-constraining measurements}

Given these examples of current Tevatron measurements that are sensitive in different ways to the description of the proton, conversely we would like to construct precision measurements with the aim of better constraining the PDF fits. The rest of this contribution will discuss several such measurements that are currently underway. 


\section{1 $\mathrm{Z}$ rapidity}

$\mathrm{Z}$ boson production is the simplest high- $p_{T}$ process at the Tevatron and has the advantage that in the clean dielectron and dimuon decay channels, the $\mathrm{Z}$ bosons are fully reconstructable. The good lepton coverage of CDF and D0 means that both experiments have access to almost the full kinematic range of $\mathrm{Z}$ production. The rapidity $y$ of the $\mathrm{Z}$ is closely related to the fractions $x_{1,2}$ of the (anti)proton momentum carried by the colliding partons, and at leading order the relation $x_{1,2}=\frac{m_{Z}}{\sqrt{s}} e^{ \pm y}$ holds exactly. A measurement of the $\mathrm{Z}$ rapidity is therefore a direct probe of the parton distributions.

$\mathrm{D} 0$ has measured the $\mathrm{Z}$ rapidity distribution in the electron channel using $337 \mathrm{pb}^{-1}$ of data, as shown in Fig. 1, and has observed good agreement with the NNLO prediction. There is potential for constraining PDFs in the high rapidity region, corresponding to extreme values of $x$ where the PDFs are less well known, with significantly increased statistics and correspondingly smaller efficiency uncertainties.
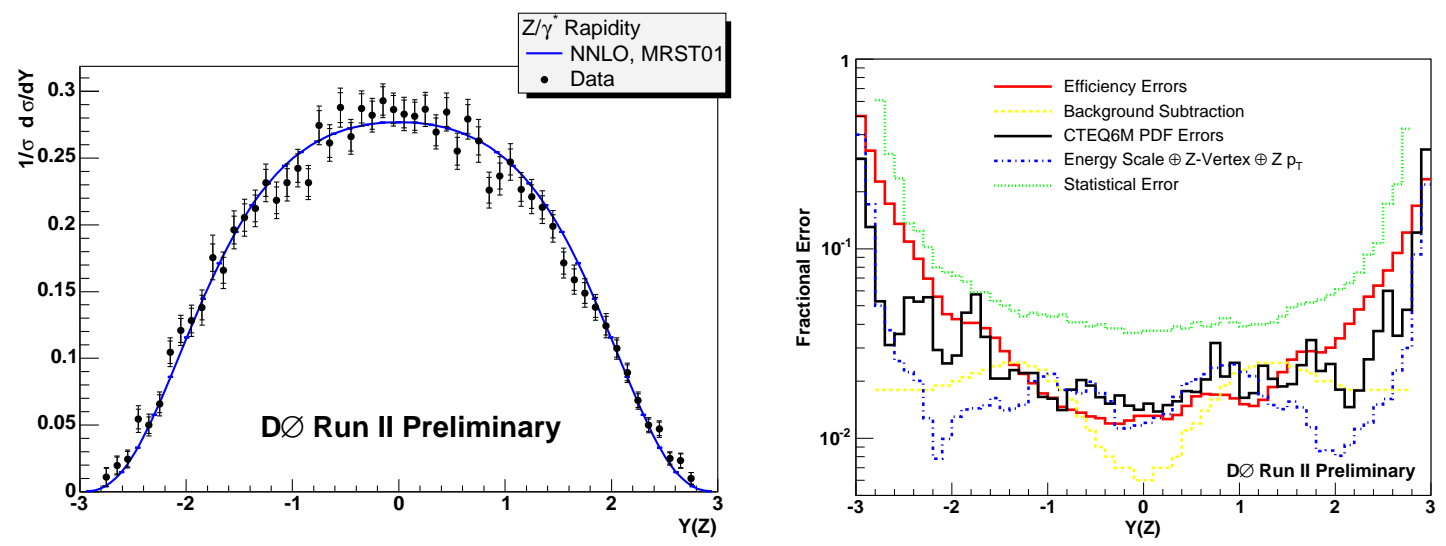

Figure 1: Left: $\mathrm{Z}$ rapidity measured by D0 in the electron channel using $337 \mathrm{pb}^{-1}$. Right: Fractional uncertainties.

\subsection{Forward W boson production}

Since the neutrino produced in the leptonic decay of a $\mathrm{W}$ boson escapes the detector, the $\mathrm{W}$ boson rapidity cannot be directly reconstructed. However as a first differential measurement of the $\mathrm{W}$ boson cross-section $\mathrm{CDF}$ has measured the cross-section for events in which the electron is reconstructed in one of the forward calorimeters, $1.2<\left|\eta_{\text {electron }}\right|<2.8$ [8]. The measurement uses $223 \mathrm{pb}^{-1}$ of data and relies on silicon-only tracking at high $\eta$. The acceptance for the central $\left(\left|\eta_{\text {electron }}\right|<1.1\right)$ and forward analyses is shown as a function of $\mathrm{W}$ rapidity in Fig 2, and it can be seen that the analyses are complementary. Also shown is the transverse mass $m_{T}=\sqrt{2 E_{T}^{\ell} E_{T}^{v}\left(1-\cos \left(\phi_{\ell v}\right)\right)}$, which demonstrates that the data are very well described and that the backgrounds are small, even far forward in the detector.

From forward events, the total $\mathrm{W}$ boson cross-section is measured to be $\sigma(\mathrm{p} \overline{\mathrm{p}} \rightarrow \mathrm{W}) \cdot \operatorname{Br}(\mathrm{W} \rightarrow$

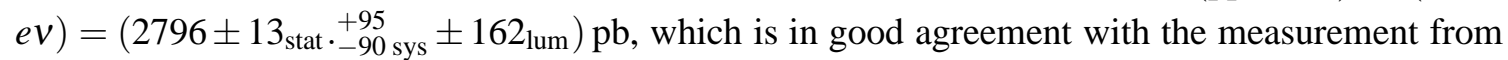
central events [5] of $\sigma(\mathrm{p} \overline{\mathrm{p}} \rightarrow \mathrm{W}) \cdot \operatorname{Br}(W \rightarrow e v)=\left(2771 \pm 14_{\text {stat }} \pm 62_{\text {sys }} \pm 166_{\text {lum }}\right) \mathrm{pb}$. 

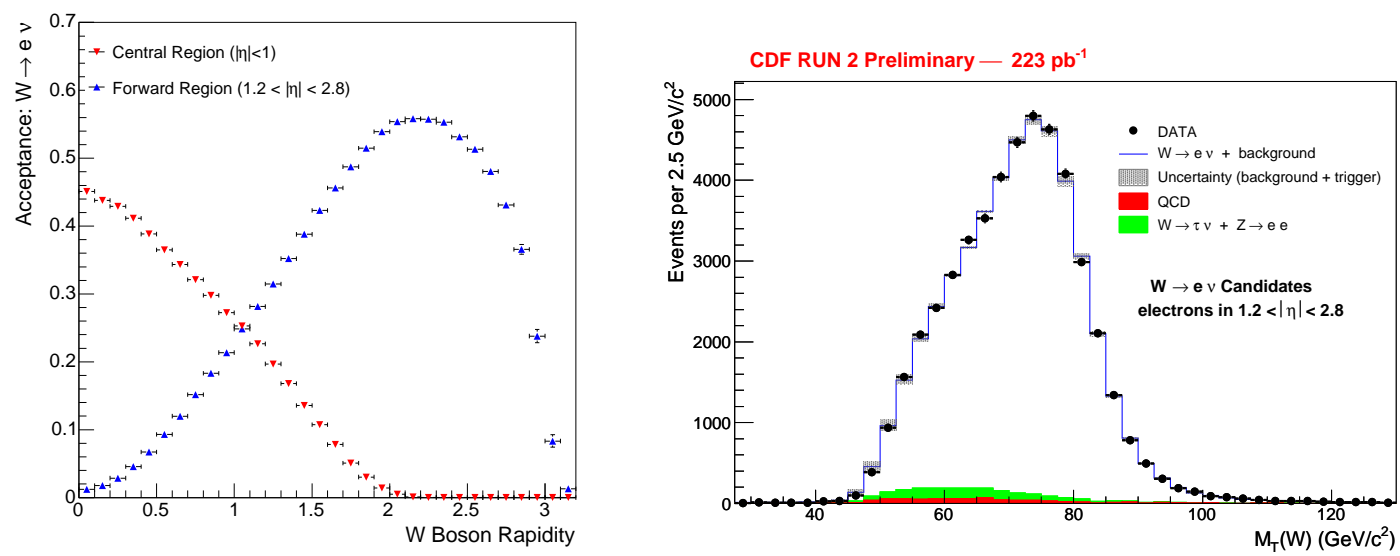

Figure 2: Left: W boson acceptance for central and forward measurements. Right: Transverse mass $m_{T}$ for forward $\mathrm{W}$ events.

The visible cross-section $\sigma_{\mathrm{vis}}$ is defined as $\sigma_{\mathrm{vis}}=\sigma_{\mathrm{total}} \cdot A$ where $A$ is the detector acceptance; then the ratio of the visible cross-sections in the central and forward regions can be compared to predictions using different PDF sets.

The analysis yields:

$$
R_{\mathrm{vis}}^{\mathrm{central} / \text { forward }}(\mathrm{CDF})=0.925 \pm 0.033
$$

and the predictions are:

$$
R_{\mathrm{vis}}^{\mathrm{cen} / \mathrm{fwd}}(\mathrm{CTEQ6.1})=0.924 \pm 0.037, R_{\mathrm{vis}}^{\mathrm{cen} / \mathrm{fwd}}(\mathrm{MRST01E})=0.941 \pm 0.012
$$

With more data, this measurement could be useful as an input to PDF fits.

\subsection{W charge asymmetry}

The rapidity is not the only handle available on $\mathrm{W}$ boson production. Since on average a higher fraction of the proton momentum is carried by u-quarks than by d-quarks, $\mathrm{W}^{+}$tend to be boosted in the proton (forward) direction and $\mathrm{W}^{-}$in the antiproton direction, resulting in a non-zero $\mathrm{W}$ charge asymmetry at a given rapidity. Experimentally the observable asymmetry is that of the decay leptons, which is diluted from the boson production asymmetry by the preferential emission of the charged lepton opposite to the boson direction in a V-A interaction.

The $\mathrm{W}$ charge asymmetry is sensitive to the ratio of $\mathrm{d}$ - to u-quarks which is otherwise not well-constrained, particularly at high $x$; and the first measurement from CDF, published in 1998 [9], changed the best-fit d-quark density by around $30 \%$ at $Q=20 \mathrm{GeV}$.

D0 has recently made its first measurement of the $\mathrm{W}$ charge asymmetry, using $230 \mathrm{pb}^{-1}$ of data in the muon channel [10]. The analysis requires an isolated track with hits in both the fibre tracker and the silicon system; and the principal systematic uncertainty is associated with the hadronic energy scale, used in the computation of the missing transverse energy associated with the neutrino. The result is given in Fig. 3 and since the experimental uncertainties are already smaller than the shaded band showing the PDF uncertainties, it should provide some constraining power. 
CDF has an earlier measurement using $170 \mathrm{pb}^{-1}$ of electron data, in which the events are separated into two bins of transverse energy: $25<E_{T}<35 \mathrm{GeV}$ and $35<E_{T}<45 \mathrm{GeV}$ [11]. Since the effect of the unknown longitudinal component of the neutrino momentum is smaller for higher $E_{T}$ electrons, more of the production asymmetry is preserved in the higher $E_{T}$ range. Furthermore, for a given $\eta_{\text {electron, }}$, the two $E_{T}$ ranges probe different $\mathrm{W}$ boson rapidities and hence different $x$. The result for the higher $E_{T}$ range is shown in Fig. 3.
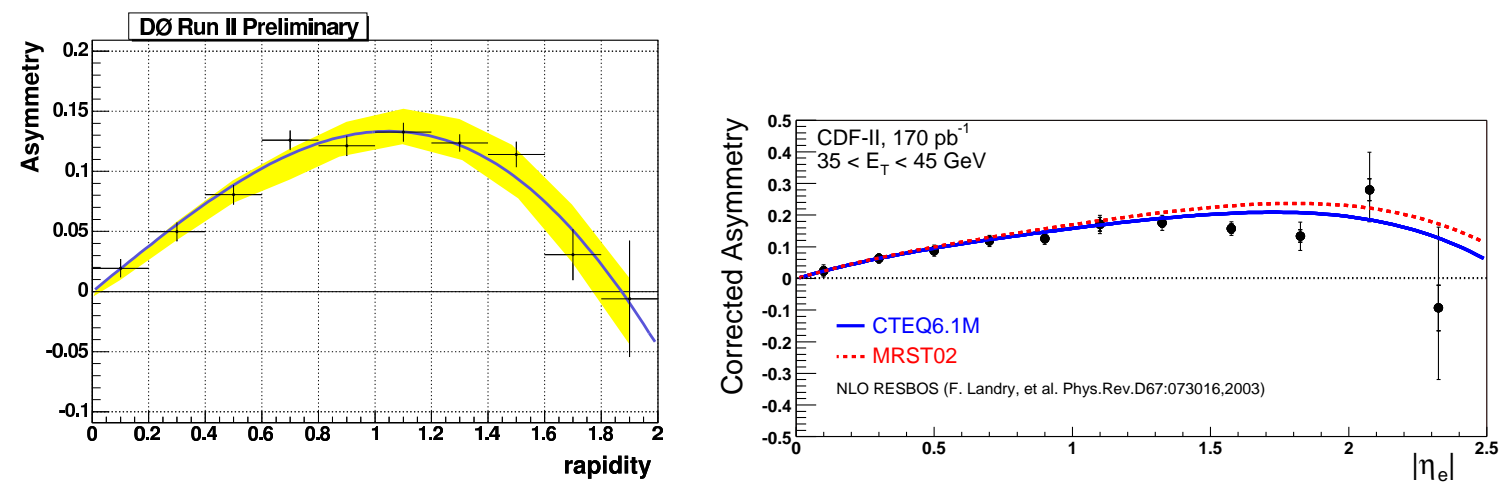

Figure 3: Left: D0 $\mathrm{W}$ charge asymmetry measurement in the muon channel, using $230 \mathrm{pb}^{-1}$. Right: CDF $\mathrm{W}$ charge asymmetry measurement in the electron channel using $170 \mathrm{pb}^{-1}$, for the high $E_{T}$ range.

CDF is currently finalising a new measurement that aims to use all of the event kinematics to unfold directly back to the $\mathrm{W}$ boson asymmetry, which will provide even further constraining power.

\subsection{Inclusive jet cross-section}

The least-constrained PDF is the gluon at high $x$, with for example the CTEQ 6.1M uncertainty reaching $100 \%$ at $x=0.6$ for $Q=500 \mathrm{GeV}$. The inclusive jet cross-section probes the gluon density directly. During Run 1 of the Tevatron CDF observed a significant excess of jets at high transverse energy, $E_{T}>300 \mathrm{GeV}$, which prompted speculation about new physics. However when proper account was taken of the uncertainty in the PDF sets used to obtain predictions, the data were found to be consistent with the Standard Model. Having highlighted the importance of correctly assessing PDF uncertainties, this measurement can now, with more statistics, be used to constrain the PDF fits.

The increase of the Tevatron's centre-of-mass energy from $1.8 \mathrm{TeV}$ to $1.96 \mathrm{TeV}$ between Run 1 and Run 2 has extended the reach of the inclusive jet cross-section by around $150 \mathrm{GeV}$ in $E_{T}$, and increased the cross-section by a factor of around three at $E_{T}=500 \mathrm{GeV}$.

Events are collected using single-jet triggers and the primary interaction is required to have taken place close to the centre of the detector, less than $50 \mathrm{~cm}$ (D0) or $60 \mathrm{~cm}$ (CDF) from the nominal interaction point. The energy in the calorimeters is summed and missing transverse energy is required to be small to remove cosmic ray and beam backgrounds.

Jets are clustered by one of two clustering methods currently in use at the Tevatron: the midpoint and $k_{T}$ algorithms. The midpoint algorithm is based on a cone in $(\eta, \phi)$-space. Cones of fixed radius $R$ are drawn around seed towers in the calorimeter, and then for improved infra-red safety 
an extra seed is placed at the midpoint in $(\eta, \phi)$ of pairs of proto-jets that are separated by less than $2 R$. Then overlapping proto-jets must be merged or split, which is determined by the energy fraction that falls in the overlap region: D0 merges if $>50 \%$ of the energy overlaps, and CDF if $>75 \%$ of the energy overlaps. The $k_{T}$ algorithm combines proto-jets according to their separation in transverse momentum $k_{T}$, starting with the smallest $k_{T}$.

The midpoint algorithm is not collinear-safe and there is some subjectivity in the merging and splitting of the proto-jets, whereas the $k_{T}$ algorithm is theoretically motivated for infra-red and collinear safety and thus for comparison with QCD predictions. However the systematic uncertainties associated with the two approaches are different and it is valuable compare the results from each.

Once jet clustering is complete, the effects of multiple proton-antiproton interactions must be removed. On average there are 1.5 inelastic $\mathrm{p}-\overline{\mathrm{p}}$ interactions per bunch-crossing, but at the highest instantaneous luminosities achieved so far, this increases to 6 interactions per crossing. D0 estimates the effect of multiple interactions by subtracting 'offset energies' measured in events requiring only crossing protons and antiprotons; and CDF subtracts energies based on the number of vertices reconstructed in an event.

Both experiments use bin-by-bin energy unfolding determined from detector simulations to reconstruct hadron-level energy distributions from the measured calorimeter-level distributions, and for its final result D0 unfolds using a parameterised functional form.

In the Monte Carlo simulation used to compare with the data, perturbative QCD partons are reconstructed into jets, and non-perturbative contributions are added from the underlying event and from fragmentation.

Both D0 and CDF have made the inclusive jet cross-section measurement in several bins of jet rapidity: D0 in two bins up to $|y|<0.8$ and CDF in five bins up to $|y|<2.1$. New physics is not expected to appear in the high rapidity bins so these can be used to constrain PDFs while maintaining sensitivity to new physics in the low rapidity bins. However the principal systematic uncertainty, the jet energy scale, is larger in the forward detectors. At CDF, a $\pm 2-3 \%$ jet energy scale uncertainty translates to a $\pm 9 \%$ cross-section uncertainty at low jet $E_{T}$ and ${ }_{-40 \%}^{+60 \%}$ at high jet $E_{T}$, and the D0 uncertainties are similar.

The results are shown in Figs. 4-6. D0's measurement (Fig. 4) uses the midpoint algorithm [12]. The data have been scaled to theory at $E_{T}=100 \mathrm{GeV}$ for $|y|<0.4$ to remove luminosity uncertainties. The shapes show excellent agreement over nine orders of magnitude of cross-section. The right-hand plot shows the experimental uncertainty band (shaded) compared to the PDF uncertainty envelope (short dash), for the higher rapidity bin.

CDF's results (Fig 5) use both the $k_{T}$ and midpoint algorithms and $1 \mathrm{fb}^{-1}$ of data [13], and show excellent agreement with predictions. The fractional uncertainties are shown for the highest rapidity bin of each analysis in Fig 6, and since the experimental uncertainties are smaller than the band allowed by the current PDF fits, these measurements will improve PDF constraints, particularly on the high- $x$ gluon.

\subsection{Z+b}

Production of $\mathrm{Z}$ bosons in association with b-quark jets receives contributions both from radiative $b$-quark generation and from the intrinsic b-quark content of the proton, the densities 

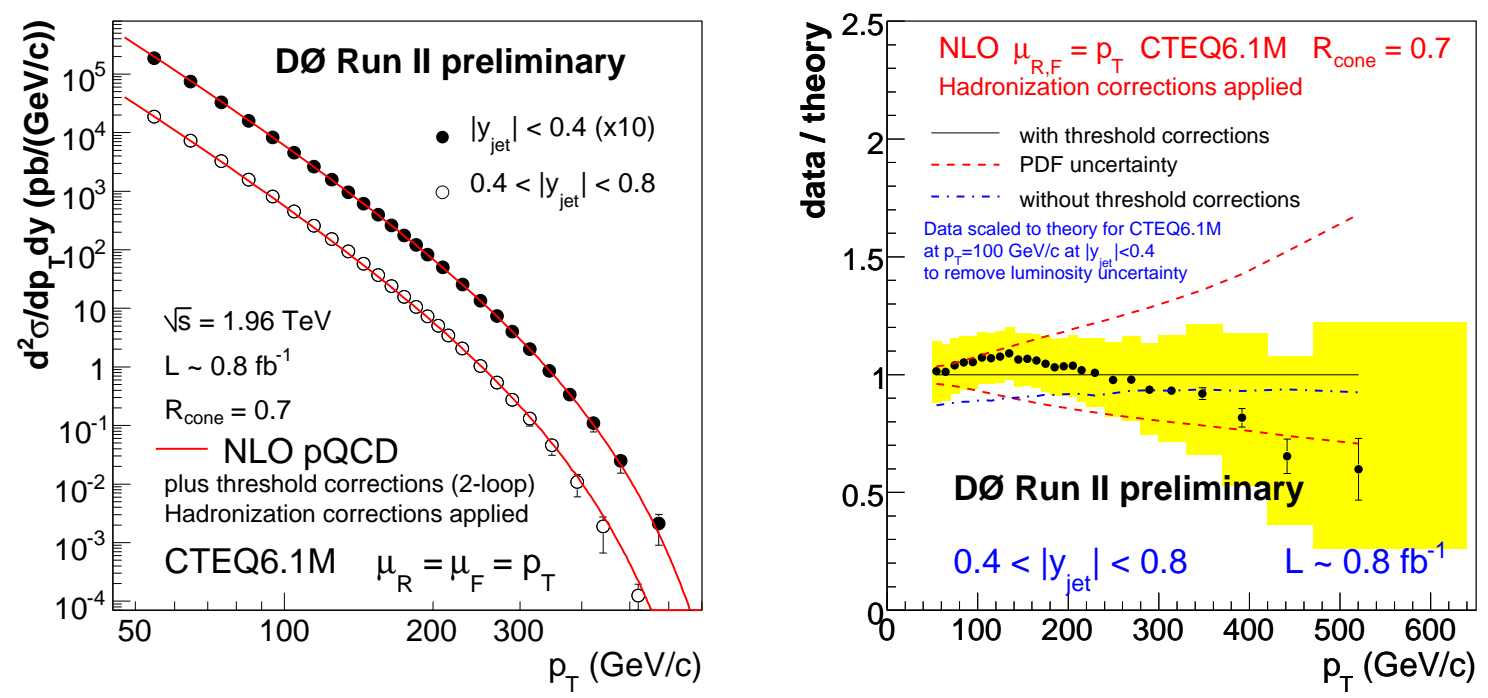

Figure 4: Left: D0 inclusive jet cross-section measurement using the midpoint clustering algorithm. Right: The ratio of measured cross-section to prediction, showing fractional uncertainties, for the higher rapidity bin.
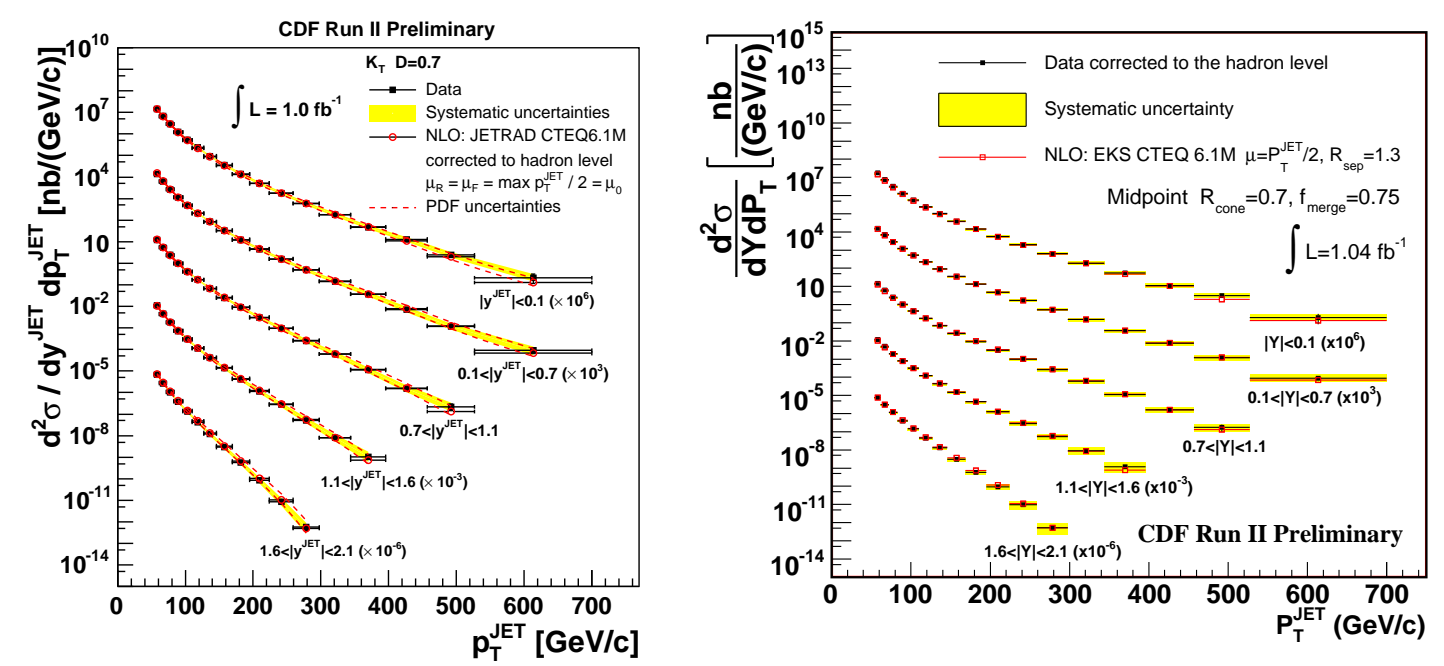

Figure 5: CDF inclusive jet cross-section measurements using (left) the $k_{T}$ clustering algorithm and (right) the midpoint clustering algorithm.

of which have only recently been measured for the first time. CDF measures the total crosssection $\sigma\left(\mathrm{Z} / \gamma^{*}+\mathrm{b}\right) \cdot \operatorname{Br}\left(\mathrm{Z} / \gamma^{*} \rightarrow\right.$ ee or $\left.\mu \mu\right)$ to be $\left(0.93 \pm 0.29_{\text {stat }} \pm 0.21_{\text {sys }}\right)$ pb. To remove luminosity uncertainties, both $\mathrm{CDF}$ and $\mathrm{D} 0$ measure of the ratio $R=\frac{\sigma(\mathrm{Z}+\mathrm{b}-\mathrm{jet})}{\sigma(\mathrm{Z} \text { jet })}$. The b-quark jets are identified by their displaced vertices relative to an event's primary vertex. CDF extracts the b-quark fraction from the reconstructed mass of all particles originating from the displaced vertex, while D0 assumes a charm content from the theoretical prediction $N_{c}=1.69 N_{b}$. The measured values are $R\left(\mathrm{CDF}, 335 \mathrm{pb}^{-1}\right)=0.024 \pm 0.007_{\text {stat }} \pm 0.005_{\text {sys }}[14]$ and $R\left(\mathrm{D} 0,180 \mathrm{pb}^{-1}\right)=$ $0.021 \pm 0.004_{\text {stat }} \cdot{ }_{-0.003}^{+0.002}$ sys $[15]$, to be compared to the NLO prediction $0.018 \pm 0.004$. Although 

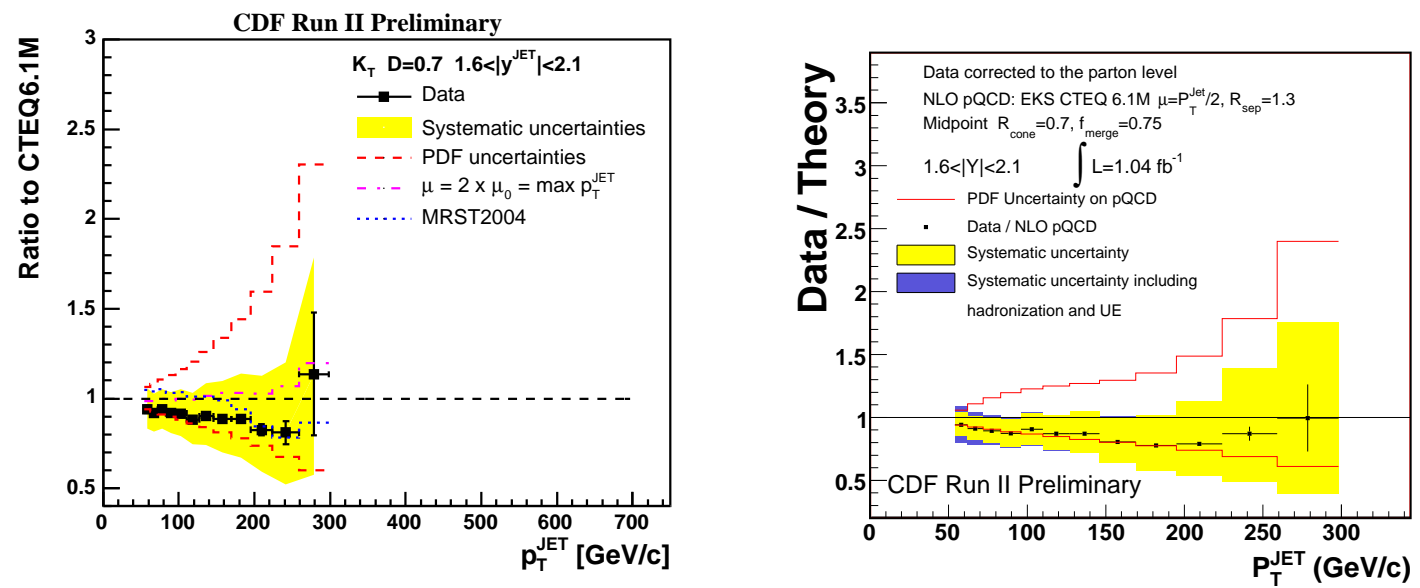

Figure 6: CDF inclusive jet cross-section fractional uncertainties in the highest jet rapidity bin, $1.6<|y|<$ 2.1, shown for (left) the $k_{T}$ clustering algorithm and (right) the midpoint algorithm.

the D0 measurement uses a smaller dataset, it quotes a smaller statistical uncertainty owing to its assumption of the charm fraction.

Further investigation of this process should lead to insights into the b-quark content of the proton, which is particularly important for searches for single-top and Higgs boson production at the Tevatron, and for many processes at the LHC.

\section{Outlook}

As the Tevatron datasets increase, PDF uncertainties become significant, entering through acceptance calculations, total theoretical cross-section predictions, template shapes and background estimates.

Both CDF and D0 have recent results for measurements that have good PDF-constraining power and are unique to the Tevatron: the $\mathrm{W}$ charge asymmetry, which gives access to the $\mathrm{d}$ quark/u-quark ratio; and the inclusive jet cross-section, which is sensitive to the high- $x$ gluon. Furthermore, several other measurements are promising for providing PDF constraints with larger datasets, such as the $\mathrm{Z}$ boson rapidity, $\mathrm{W}$ bosons produced in the forward region, and $\mathrm{Z}$ bosons produced in association with b-quarks. 


\section{Acknowledgments}

The author would like to thank Glasgow University and the UK Particle Physics and Astronomy Research Council for financial support.

\section{References}

[1] http://www.phys.psu.edu/ cteq

[2] http://www-spires.dur.ac.uk/hepdata/mrs.html

[3] J. Pumplin et al., JHEP 07 (2002) 012.

[4] A. D. Martin et al., Phys. Lett. B604 (2004) 61; Eur. Phys. J. C28 (2003) 455.

[5] D. Acosta et al. (CDF Collaboration), Phys. Rev. Lett. 94 (2005) 091803.

[6] CDF Collaboration, CDF Conference Note 8148 (2006).

[7] M. Cacciari et al., JHEP 04 (2004) 068.

[8] CDF Collaboration, CDF Conference Note 8119 (2006).

[9] F. Abe et al. (CDF Collaboration), Phys. Rev. Lett. 81 (1998) 5754.

[10] D0 Collaboration, D0 Conference Note 5061 (2006).

[11] D. Acosta et al. (CDF Collaboration), Phys. Rev. D 71 (2005) 052002.

[12] D0 Collaboration, D0 Conference Note 5087 (2006).

[13] CDF Collaboration, CDF Conference Notes 8374, 8388 (2006).

[14] A. Abulenia et al. (CDF Collaboration), Phys. Rev. D 74 (2006) 032008.

[15] V. M. Abazov et al. (D0 Collaboration), Phys. Rev. Lett. 94 (2005) 161801. 